\title{
Modeling biomarker dynamics with implications for the treatment of prostate cancer
}

\author{
ERIN B. HEDICAN†, JOHN T. KEMPER $+*$ and NICOLE M. LANIEף \\ $†$ School of Public health, University of Minnesota, Minneapolis, MN 55155, USA \\ ‡Department of Mathematics, University of Saint Thomas, Saint Paul, MN 55105, USA \\ ๆ Department of Mathematics, Western Michigan University, Kalamazoo, MI 49008, USA
}

(Received 10 November 2005; revised 28 February 2007; in final form 13 March 2007)

\begin{abstract}
The authors review existing models of biomarker dynamics and develop and investigate several new models which may better accommodate the underlying biology. While the general foundations of the models studied could be applied to a number of biomarker systems, the parameter values and specific applications to treatment regimens are focused on the role of prostate-specific antigen (PSA) as a biomarker for prostate cancer. Included are suggestions for possible clinical validation studies.
\end{abstract}

Keywords: Prostate-specific antigen; Prostatic intraepithilial neoplasia; Benign prostate hyperplasia; Biomarker dynamics; Biomarker models

\section{Introduction}

A human biomarker system provides evidence of the relationship between a naturally occurring and generally harmless or even beneficial biological product (often an enzyme) that can be observed without serious physical insult to the human subject and an underlying disease or condition (for example, cancer), the direct observation of which requires higher-risk, invasive methods. By monitoring the level of the biomarker, clinicians expect to better understand and anticipate changes in the underlying condition, in order to suggest when more invasive testing or treatment should be scheduled most efficiently. While biomarkers have been identified in connection with such disorders as Alzheimer's disease, exposure to toxic agents and several cancers, the one known best is probably PSA [1-6].

Prostate-specific antigen (PSA) is a proteolytic enzyme produced by epithelial prostate cells that serves to liquefy seminal fluid [7]. Under the regulatory control of male hormones, newly synthesized PSA is secreted into the prostatic ducts and concentrated in the seminal fluid. Most of the PSA produced by these prostate cells is naturally removed from the body through the semen, but small amounts of the protein are able to diffuse into the circulation and can be measured in the bloodstream [8]. Normally, leakage of PSA into the blood is controlled by natural barriers between the prostatic ducts and circulation that set up a million-fold concentration difference between the prostate fluid and the plasma. These barriers include intact cellular tight junctions connecting neighboring epithelial cells, an

*Corresponding author. Email: jtkemper@stthomas.edu 
undifferentiated basal cell layer, the basement membrane, and the stroma $[9,10]$. To enter the circulation, PSA must cross through these tissue layers and then pass into the blood vessels, where the antigen either exists in the free form or is inactivated and controlled by irreversible binding to protease inhibitors in the blood [8]. Disruption or breakdown of these barriers has been found to accompany prostatic intraepithilial neoplasia (PIN), a precursor to prostate cancer [11,12]. A comprehensive review of prostate pathology can be found in Ref. [13]. The potential degrading of these barriers by tumor cells and the resulting effect on biomarker levels is one of the principal considerations in this study.

Normal values of serum PSA are considered to be at or below $4 \mathrm{ng} / \mathrm{ml}$, but there are many conditions that may prompt an elevated level of serum PSA [6], including prostatic infection, recent biopsy of the prostate, benign prostate hyperplasia (BPH), and prostate cancer [4]. Among these, adenocarcinoma of the prostate has been found to lead to the greatest increase of serum PSA, often producing levels that are 10-times greater per gram of tissue than those seen in normal or benign conditions [4]. Due to these findings, PSA has been used as a biomarker to diagnose and monitor prostate cancer, with an increase of PSA acting as an indicator for an increased number of cancerous cells in the prostate gland.

That rising levels of PSA in the bloodstream may result, in part, from the disruption of natural barriers that separate the contents of the affected organ from circulation has been a conclusion reached in several studies $[14,9,10]$. Prostate cancer cells do not produce more PSA on a per cell basis than benign or normal tissues but rather increase the leakage of PSA across the barriers and into the bloodstream $[9,8]$. The fast replication rate of the cancer cells leads to increased cell numbers situated in dense populations which are able to destroy the organization of the normal epithelial cells and infiltrate the bloodstream. The increase in PSA found in the serum of prostate cancer patients is thus due not only to an increase in the number of cells producing PSA, but also to the breakdown in the barriers affected by the underlying disease.

One of the principal uses of the biomarker models reviewed and developed below is to support and monitor treatment of the disease condition. Although multiple treatments are presently available, the primary method of cancer treatment considered in this analysis is radiotherapy. The two forms of radiation treatment that exist for the control of prostate cancer, external beam radiation therapy and brachytherapy, both act at the cellular level, causing extensive damage to the DNA within a cell, resulting in the loss of reproductive capability and ultimately in cell death [9]. The goal of radiation therapy is to administer a large enough dose to the targeted organ to destroy the cancerous cells while maintaining sufficient normal cells and surrounding tissue to allow for continued successful organ function $[15,16,9]$.

In formulating a model that accurately reflects the biology and kinetics of cancer growth and treatment, there are many factors that must be taken into consideration. Previous models $[17,18]$ reflected the exponential growth of tumor cells and the decay of treatment effectiveness over time, but have not directly considered the mechanism by which the increase in biomarker is related to tumor growth. Models that describe this relationship in greater detail may allow researchers to better determine optimal radiation dosages and better estimate parameters associated with the disease and its response to treatment. Such models, particularly the system models, are developed below with emphasis on the extent to which the model dynamics express qualitative features of the underlying biology. In several instances, clinical experiments are suggested as a step toward further model validation. 


\section{Simulation of treatment dynamics: a basic model}

Any model which simulates the behavior of a biomarker in response to treatment of the underlying disease or condition must accurately represent the three fundamentally different treatment outcomes. The treatment can be successful, resulting in complete elimination or sustained control of the disease. Otherwise, the treatment may appear to be successful at first, with a subsequent relapse of disease or can simply be ineffective from the outset. A basic deterministic model proposed for radiation treatment of prostate cancer (see references in Ref. [17]) is based on the ordinary differential equation

$$
\frac{\mathrm{d} x}{\mathrm{~d} t}=\alpha x(t)-k \mathrm{e}^{-a t}
$$

for the evolution of PSA level, $x$, with respect to time, $t$. PSA levels are measured in $\mathrm{ng} / \mathrm{ml}$ and time is measured in days. Parameters represent the growth rate of cancer, $\alpha$, the decay rate of treatment, $a$, and the level or intensity of radiation treatment, $k$. Equation (1) is subject to the initial condition

$$
x(0)=x_{0}
$$

where $x_{0}$ is the PSA level at time $t=0$. The solution to (1) and (2) is well known to be

$$
x(t)=\frac{k}{\alpha+a} \mathrm{e}^{-a t}+\left(x_{0}-\frac{k}{\alpha+a}\right) \mathrm{e}^{\alpha t} .
$$

Parameters for this "bi-exponential" model can be selected to simulate each of the three treatment scenarios. As long as $\alpha>a$ (tumor growth is slow relative to the decay rate of treatment) and $k>x_{0}(\alpha+a)$ (treatment dosage is sufficiently high), the treatment is successful, but the model in this case leads to negative levels of the biomarker, an impossibility. If $k>x_{0}(\alpha+a)$ but $\alpha<a$, the treatment success is only temporary (until $t=1 /(\alpha+a) \ln \left(a k / \alpha\left(k-x_{0}(\alpha+a)\right)\right)$, while if $k<x_{0}(\alpha+a)$, the treatment fails from the outset. In addition to the problem of allowing negative biomarker levels, this model incorporates at least one (related) questionable biological assumption, namely that the effect of treatment on biomarker levels (and, by implication, on tumor mass) is unrelated to the biomarker level (tumor mass) itself. Despite these shortcomings this model was found to be useful in several studies [19-21].

\section{Parameter selection issues}

In a specific application of (3) above, parameter values used must be based on biological data. These values can be chosen to match individual circumstances and may be different for different patients or different treatment protocols.

Following successful radiation treatment of prostate cancer, clinicians report PSA values of $0.5-1.0 \mathrm{ng} / \mathrm{ml}$ after 17 months, reflecting a very small probability of relapse in a patient's lifetime [17]. Values for $x_{0}$, the pre-treatment PSA level, typically vary in the range of $4.0-20.0 \mathrm{ng} / \mathrm{ml}$. (Even for patients with initial PSA readings below this range, an increase in PSA of $0.75 \mathrm{ng} / \mathrm{ml}$ or greater over a year-long period is an indicator that cancer should 
be considered [4].) For simulations based on (3), values for $x_{0}$ are chosen in the range of $4.7-5.0 \mathrm{ng} / \mathrm{ml}$.

One measurement of the doubling time of prostatic tumors is 67 days, which gives a value for $\alpha$ to be 0.01 day $^{-1}[22,8]$. The treatment decay rate used in this model comes from the brachytherapy half-lives of the two isotopes commonly used for the internal treatment of prostate cancer, $\mathrm{Pd}^{103}$ and $\mathrm{I}^{125}$. The average half-life of these two isotopes is about 30 days, yielding the value of 0.02 day $^{-1}$ for $a[16,23]$.

The parameter for treatment intensity, $k$, in the model is required to have the units of day $^{-1}$. In clinical situations, treatment intensity or dose is measured in Grays (Gy), a value which corresponds to one joule per kilogram of tissue [9]. In this model, however, this parameter has a value equal to the fraction per unit time of cells killed by radiation when it is at its most effective level. Radiation treatment doses are typically given to prostate cancer patients once every $24 \mathrm{~h}$ to allow the normal cells, but not the less effectively repairing cancerous cells, to recover from the induced damage [16]. Based on this daily regimen, the half-life for treated cells is taken to be 1 day in the simulation, with the corresponding value of $k=0.069$ day $^{-1}$ [25].

The biomarker dynamics derived from (3) using parameter values associated with PSA as described above show dramatically negative values of PSA as time increases. While this model may accurately approximate observed biological behavior over a short time, model outcomes with negative biomarker levels are a clear indication of a deficiency. Besides this and the fact that treatment effectiveness is independent of tumor size, the model (3) does not allow for non-constant yet stable patterns of biomarker behavior in the absence of disease and treatment.

\section{Alternative single-equation models}

An alternative version of the model derived from (1) which does accommodate a stable baseline level of biomarker, $b$, is represented by

$$
\frac{\mathrm{d} x}{\mathrm{~d} t}=\alpha(x(t)-b)-k \mathrm{e}^{-a t},
$$

where only the levels of PSA that are above the baseline are reflective of the growing cancer. The initial value of PSA just prior to treatment is generally above the baseline. The solution to the model based on (4) and (2) is found, using standard methods, to be

$$
x(t)=\frac{k}{\alpha+a} \mathrm{e}^{-a t}+\left(x_{0}-\frac{k}{\alpha+a}-b\right) \mathrm{e}^{\alpha t}+b .
$$

With the same parameter values (derived for PSA) that were described above, along with a value of $b=4 \mathrm{ng} / \mathrm{ml}$, the new model (5) is similar to (3) in that it also produces unrealistic negative values of PSA and assumes a treatment impact independent of disease level.

In a third model given by differential equation (6) below, we modify the treatment term to reflect the fact that treatment effectiveness applies only to disease, represented by the extent to which the biomarker level exceeds its baseline. This new differential 
equation is

$$
\frac{\mathrm{d} x}{\mathrm{~d} t}=\alpha(x(t)-b)-k\left(\frac{x(t)}{b}-1\right) \mathrm{e}^{-a t}
$$

again paired with the initial condition (2). Standard methods determine the solution in this case to be

$$
x(t)=b+\mathrm{e}^{(k / a b)\left(\mathrm{e}^{-a t}-1\right)+\alpha t}\left(x_{0}-b\right),
$$

with a choice of parameters identical to those used for equation (4).

Of these first three models, (6) most closely matches the experience generally observed, depicting treatment success in the near term with a relapse after about 200 days (figure 1). For certain parameter values, the relapse will be postponed beyond a patient's life expectancy, thus resulting in what appears to be a complete cure. For initial levels of biomarker greater than the baseline level, the lowest possible subsequent level is at the baseline. However, in many cases of radiation treatment for prostate cancer, the lowest level seen after treatment is less than $4.0 \mathrm{ng} / \mathrm{ml}$, and values in the range of $0.5-1.0 \mathrm{ng} / \mathrm{ml}$ are sometimes used as indicators of treatment success [7,8]. To address this apparent shortcoming, one might adjust downward the baseline to a post-treatment baseline. With this adjustment, the model (6) provides a more realistic picture of likely biomarker dynamics, with the reservation that still not all biological implications (e.g. PSA loss rate) are considered. Another concern is that an initially low biomarker level $\left(x_{0}<b\right)$ eventually results again in unrealistic negative levels.

Our fourth model, expressed by differential equation (8), captures the idea that the level of treatment effect should be proportional to the amount or volume of cancer cells being treated. Accordingly, we have modified (1) to include the biomarker variable $x(t)$ in both the treatment and decay terms, obtaining

$$
\frac{\mathrm{d} x}{\mathrm{~d} t}=\alpha x(t)-k x(t) \mathrm{e}^{-a t} .
$$

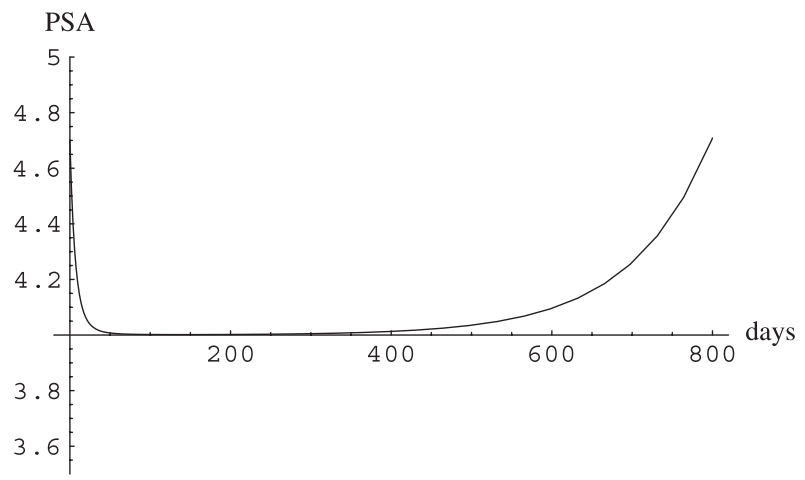

Figure 1. This figure gives the modeled course of PSA levels as described by (6) with parameter values $b=4.0$, $a=0.02, k=0.639, \alpha=0.01, x_{0}=4.7$. Note the long period of disease control. 
This equation is also subject to the initial condition (2) and can then be solved using standard methods to obtain

$$
x(t)=x_{0} \mathrm{e}^{(\alpha t+(k / a))\left(\mathrm{e}^{-a t}-1\right)} .
$$

Although a baseline biomarker level is not involved here in either treatment or growth terms, this model is valuable in that it allows both long-term cure and eventual relapse, with no possibility of negative biomarker values. Considering that most men diagnosed with prostate cancer are over the age of 50, it is not uncommon for male patients to die with an undetected cancerous mass residing in the prostate $[4,6]$, suggesting a potential for relapse that may never be realized. After 5 years, if PSA has not shown more than three consecutive and detectable increases, current treatment evaluation protocols may determine that the treatment is a success [4].

Each of the single-equation models may have appropriate uses over certain ranges of parameter values and over short time periods, but none are fully satisfactory in allowing the variety of biomarker behavior noted in clinical studies and sometimes including high-low fluctuations. In addition, despite the positive features of the model with solution (9), there is no identified level of disease (tumor size) associated with a particular biomarker level and the model allows only constant solutions in the absence of disease and treatment.

\section{System models for biomarker dynamics}

We will now consider systems of differential equations that address some of the inadequacies of the single-equation models for biomarker dynamics. These models take into account that normal cells may also contribute to the level of biomarker. In addition, the natural process for removal of biomarker from the system will be modeled explicitly, as will the effect of tumor cells on the integrity of the organ membranes. While single-equation models necessarily combine characteristics of the biomarker and the underlying disease organism, the system models identify as separate entities the biomarker, $x(t)$, the diseased functioning cells, $y(t)$, and the normal functioning cells, $z(t)$.

A basic version of the system model is represented by equations

$$
\begin{gathered}
\frac{\mathrm{d} x}{\mathrm{~d} t}=-\gamma x(t)+\mu(y(t)+z(t)) \\
\frac{\mathrm{d} y}{\mathrm{~d} t}=\alpha y(t)-k \mathrm{e}^{-a t} y(t) \\
\frac{\mathrm{d} z}{\mathrm{~d} t}=0
\end{gathered}
$$

with initial condition $x(0)=x_{0}, y(0)=y_{0}$ and $z(0)=z_{0}$.

This model reflects the fact that biomarker levels are increased equally (at a rate $\mu$ that is independent of disease level) by normal and diseased cells (as is true in prostate cancer) and decreased through elimination from the serum (at rate $\gamma$ ). The level of diseased cells is influenced by the growth rate of diseased cells $\alpha$, and the treatment level $k$, assumed in this model to decay exponentially as would be expected for a radioactive 
seed. In this first system model, the normal cell population level is taken to be constant. It is easily seen that $z(t)=z_{0}$ and

$$
y(t)=y_{0} \mathrm{e}^{(\alpha t-(k / a))\left(1-\mathrm{e}^{-a t}\right)}
$$

so that the biomarker equation can be solved to yield

$$
x(t)=\mathrm{e}^{-\gamma t}\left(x_{0}+\mu \int_{0}^{t} \mathrm{e}^{-(k / a)+\gamma s}\left(y_{0} \mathrm{e}^{\alpha s+(k / a) \mathrm{e}^{-a s}}+z_{0} \mathrm{e}^{(k / a)}\right) \mathrm{d} s\right) .
$$

In the absence of disease $\left(y_{0}=0\right)$ and treatment $(k=0)$, the biomarker level is $x(t)=\left(\mu z_{0} / \gamma\right)+\left(x_{0}-\left(\mu z_{0} / \gamma\right)\right) \mathrm{e}^{-\gamma t}$, rapidly approaching the stable value $\mu z_{0} / \gamma$. In the case of untreated disease, $x(t)=\left(\mu z_{0} / \gamma\right)+\left(\mu y_{0} /(\alpha+\gamma)\right) \mathrm{e}^{\alpha t}+\left(x_{0}-\mu\left(y_{0} /(\alpha+\gamma)\right)+\right.$ $\left.\left(z_{0} / \gamma\right)\right) \mathrm{e}^{-\gamma t}$, showing a biomarker increase at the same (ultimate) rate of growth as that of the diseased cells. (This connection may provide clinicians with a non-invasive method to confirm tumor growth rates.)

For an application to PSA, reasonable initial and parameter values are $x_{0}=5.0 \mathrm{ng} / \mathrm{ml}$ [6], $y_{0}=1.0 \mathrm{~cm}^{3}$ [10], $z_{0}=40 \mathrm{~cm}^{3}$ [24], $\gamma=0.3 \mathrm{day}^{-1}$ [9], $\alpha=0.01 \mathrm{day}^{-1}$ [22,8], $a=0.02$ day $^{-1}[16]$, and $k=0.07$ day $^{-1}$ [25]. The "transport rate" $\mu$, the rate at which PSA "leaks" into the serum, has only recently become an object of study, but its value can be taken from the no-disease case to be $\mu=0.04(\mathrm{ng} / \mathrm{ml}) \mathrm{cm}^{-3}$ day $^{-1}$. For these initial parameter values, the evolving PSA level, determined by (14), gives evidence of an early peak around day 10 , followed by a long period of gradual biomarker decline, with a relapse indicated roughly two years later. By contrast, there are no preliminary peaks in the patterns observed for the singleequation models (following a single treatment). Because clinicians have observed such fluctuations in individual cases [4], the system model suggests a more realistic simulation of the biomarker dynamics.

Other advantages of the model represented by (10)-(12) over the bi-exponential model represented by (1) are that it distinguishes between the biomarker and the underlying disease, that it accommodates the leakage of biomarker into the serum and that it expresses treatment success proportional to the amount of diseased tissue (tumor size), thereby not allowing any of the variables to become negative. However, this model is limited by the assumption that the rate of leakage is constant. PSA studies such as those referred to in Ref. [14,9], suggest that, while the production of PSA occurs at the same rate in normal and cancerous prostate cells, the cancer cells have the ability to degrade the prostate epithelial cells and other barriers that normally restrict the flow of PSA out of the gland. Another limitation results from the assumption of constant normal tissue volume.

To address the limitation in the system model related to leakage rates, we replace the constant leakage (or transport) rate $\mu$ in (10) with a functional $\mu(y(t))$ to express the dependence of the leakage on level of disease. We will consider first a linear form of $\mu(y)$ : $\mu(y)=A y+B$. Of course, there have been few opportunities yet for clinicians to investigate the nature of leakage, so a linear form should only be considered an approximation until future studies can confirm details of the membrane degradation process and its effects. Other functional forms that might prove useful include, for example, fractional powers to characterize an effect only on the tumor surface or a sigmoidal form which ranges between specific low and high values. In the linear case, (10) is replaced by

$$
\frac{\mathrm{d} x}{\mathrm{~d} t}=-\gamma x(t)+(A y(t)+B)(y(t)+z(t)) .
$$


Using the solutions for $y(t)$ and $z(t)$ already found, the biomarker equation becomes

$$
\frac{\mathrm{d} x}{\mathrm{~d} t}=-\gamma x(t)+\left(A y_{0} \mathrm{e}^{\alpha t-(k / a)\left(1-\mathrm{e}^{-a t}\right)}+B\right)\left(y_{0} \mathrm{e}^{\alpha t-(k / a)\left(1-\mathrm{e}^{-a t}\right)}+z_{0}\right)
$$

with solution

$$
x(t)=\mathrm{e}^{-\gamma t}\left(x_{0}+\int_{0}^{t} \mathrm{e}^{\gamma s-(2 k / a)\left(1-\mathrm{e}^{-a s}\right)}\left(A y_{0} \mathrm{e}^{\alpha s}+B \mathrm{e}^{(k / a)\left(1-\mathrm{e}^{-a s}\right)}\right)\left(y_{0} \mathrm{e}^{\alpha s}+z_{0} \mathrm{e}^{(k / a)\left(1-\mathrm{e}^{-a s}\right)}\right) \mathrm{d} s\right) .
$$

From the discussion of parameter values for PSA levels following (14), a reasonable value for $B$ is $0.04(\mathrm{ng} / \mathrm{ml}) \mathrm{cm}^{-3}$ day $^{-1}$. More clinical evidence is required for a reliable value for $A$, but a plausible value is $A=0.04(\mathrm{ng} / \mathrm{ml}) \mathrm{cm}^{-6}$ day $^{-1}$, suggesting a doubling of leakage as tumor size grows from 1.0 to $2.0 \mathrm{~cm}^{3}$. With the other parameter values as determined above, the result of simulation using (15) can be seen in figure 2, with a pronounced preliminary peak in biomarker level at about five days post-treatment and relapse indicated at around 400 days. The fact that the model may be calibrated to replicate the early peak and then used to forecast the later time of relapse may be helpful in scheduling follow-up testing and possible treatment. Clinical evidence might also be sought to validate the temporal pattern predicted by the model.

To address the limitation related to the assumption of constant volume of normal tissue, we will incorporate into the system model two additional realistic assumptions. The first is that the treatment may affect normal cells as well as diseased cells, although the impact on normal cells is typically less because the cell repair mechanism has not been compromised and/or the treatment may be targeted more directly at diseased cells. The second new assumption is that normal cell volume is regulated through a logistic mechanism with a predetermined (for each individual) equilibrium size $z_{1}$. With these additional assumptions
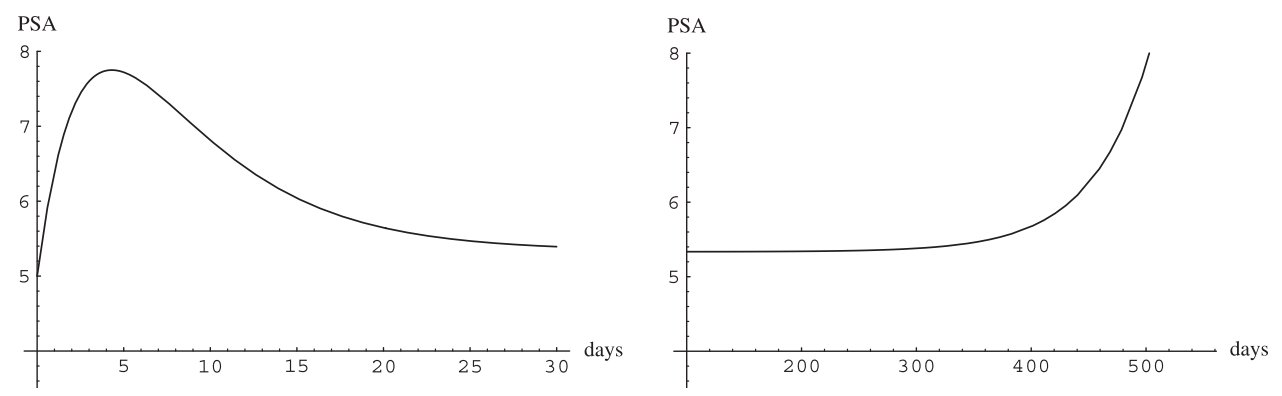

Figure 2. Evolution of PSA modeled from (15) with parameter values $A=0.04, B=0.04, a=0.02, k=0.07$, $\mu=0.04, \gamma=0.03, \alpha=0.01, x_{0}=5, y_{0}=1$ and $z_{0}=40$, over a time span of 30 days. Note the early peak in biomarker level followed by a long decline. The second panel shows the continuation of PSA levels with identical parameter values over a span of 100-550 days, indicating a likely tumor resurgence after about 440 days. 
the equations governing the complete system model can be expressed as

$$
\begin{gathered}
\frac{\mathrm{d} x}{\mathrm{~d} t}=-\gamma x(t)+\mu(y(t))(y(t)+z(t)) \\
\frac{\mathrm{d} y}{\mathrm{~d} t}=\alpha y(t)-k_{y} \mathrm{e}^{-a t} y(t) \\
\frac{\mathrm{d} z}{\mathrm{~d} t}=r z(t)\left(1-\frac{z(t)}{z_{1}}\right)-k_{z} \mathrm{e}^{-a t} z(t)
\end{gathered}
$$

where the transport function $\mu(y)$ is a smooth nondecreasing function with $\lim _{y \rightarrow \infty} \mu(y) \geq \mu(0)>0$. The two cases treated earlier for constant $z(t)$ were cases with $\mu(y)=\mu$ (constant) and $\mu(y)=A y+B$ (linear).

The solution of (20) with initial condition $z_{0}=z_{1}$ is given by

$$
z(t)=\frac{z_{1} \mathrm{e}^{r t+\left(k_{z} / a\right) \mathrm{e}^{-a t}}}{\mathrm{e}^{\left(k_{z} / a\right)}+r \int_{0}^{t} \mathrm{e}^{r s+\left(k_{z} / a\right) \mathrm{e}^{-a s}} \mathrm{~d} s}
$$

With $z(t)$ given by $(21)$ and $y(t)$ given by (13) with $k_{y}$ in place of $k$, the form of the biomarker equation (18) becomes

$$
x^{\prime}(t)=-\gamma x(t)+\mu\left(y_{0} \mathrm{e}^{\alpha t-\left(k_{y} / a\right)\left(1-\mathrm{e}^{-a t}\right)}\right)\left(y_{0} \mathrm{e}^{\alpha t-\left(k_{y} / a\right)\left(1-\mathrm{e}^{-a t}\right)}+\frac{z_{1} \mathrm{e}^{r t+\left(k_{z} / a\right) \mathrm{e}^{-a t}}}{\mathrm{e}^{\left(k_{z} / a\right)}+r \int_{0}^{t} \mathrm{e}^{r s+\left(k_{z} / a\right) \mathrm{e}^{-a s} \mathrm{~d} s}}\right),
$$

which has the solution given by (22) in Remark 2 below.

In the case of prostate cancer, as mentioned above, the elevated levels that are characteristic of disease are prompted by the breakdown of natural barriers separating the contents of the prostatic epithelial lumen from the bloodstream [14,9,10], allowing biomarkers produced by both diseased and normal cells to enter the bloodstream more easily. The mechanism for the disruption of barriers and subsequent increase in serum PSA is accounted for in the model by the transport term $\mu(y)$. Future investigations of the membrane degradation process may contribute to a better understanding of the transport phenomenon. Analogous transport processes may play a role in other biomarker systems. When values for $B \equiv \mu(0)$ and $C \equiv \lim _{y \rightarrow \infty} \mu(y)$ can be estimated, along with the level of disease $y=D$ where $\mu(y)$ is increasing most rapidly, one appealing form of the transport function is $\mu(y)=(B+C) / 2+(C-B) / 2 \operatorname{erf}(\omega(y-D))$ where $\omega$ determines the overall rate at which transport across the barrier takes place and $\operatorname{erf}(\cdot)$ is the standard error function. Clinical studies may be helpful in identifying parameter values if this particular form is adopted.

A comprehensive examination of the possible scenarios included in the model (18)-(20) is provided by the following result:

Theorem (Qualitative Behavior of Disease/Biomarker Dynamics). Let $x(t), y(t)$ and $z(t)$ be the levels of biomarker, diseased tissue and normal tissue, respectively, satisfying equations (18)-(20) where parameters identify the rate of removal of biomarker from the 
body, $\gamma$, the growth rate of disease/tumor, $\alpha$, the intensity of initial treatment on diseased cells, $k_{y}$, the intensity of treatment on normal cells, $k_{z}$, the rate at which treatment effectiveness decays, $a$, and the rate at which normal tissue "relaxes" to its equilibrium level, $r$, and where the transport function $\mu(\cdot)$ is a smooth non-decreasing function on $[0, \infty)$ with $\mu(0)>0$ and $\lim _{y->\infty} \mu(y)<\infty$. Initial values $x(0)=x_{0}, y(0)=y_{0}$ and $z(0)=z_{0}$ are non-negative and $x_{1}=\mu(0) z_{1} / \gamma$ and $z_{1}$ are positive (non-disease) equilibrium levels of the biomarker and normal tissue, respectively. Then the following dynamics are possible.

Case A (no disease, no treatment). If $y_{0}=0$ and $k_{y}=k_{z}=0$, then one of the following occurs:

(A1) $z(t) \equiv z_{1}$ and $x(t)$ approaches $x_{1}$ monotonically, or

(A2) $z(t)$ approaches $z_{1}$ monotonically and, after at most one critical point, $x(t)$ approaches $x_{1}$ monotonically.

Case B (treatment, no disease). If $y_{0}=0$ with $k_{y}>0$ (and $k_{z} \geq 0$ ), then one of the following occurs:

(B1) $z(t)$ increases monotonically to $z_{1}$ and, after at most one local minimum, $x(t)$ increases monotonically to $x_{1}$, or

(B2) $z(t)$ first decreases to a minimum value $z_{\min }<z_{1}$ and then increases monotonically to $z_{1}$ while $x(t)$ may experience a single maximum (while $z^{\prime}(t)<0$ ), possibly followed by a single minimum (while $z^{\prime}(t)>0$ ), after which $x(t)$ approaches $x_{1}$ monotonically.

Case $C$ (disease, no treatment). If $y_{0}>0$ and $k_{y}=k_{z}=0$, then one of the following occurs:

(C1) $z(t) \equiv z_{1}, y(t)$ increases without bound and, possibly following a single local minimum, $x(t)$ increases without bound, or

(C2) $z(t)$ approaches $z_{1}$ monotonically, $y(t)$ increases without bound and, $x(t)$ may experience at most one local minimum, possibly preceded by at most one local maximum, before increasing without bound.

Case $D$ (disease and treatment). If $y_{0}>0, k_{y}>0$ and $k_{z}>0$, then one of the following occurs:

(D1) $z(t)$ increases monotonically to $z_{1}$ (if $\left.k_{z} \leq r\left(1-\left(z_{0} / z_{1}\right)\right)\right)$ ), $y(t)$ increases without bound (if $k_{y} \leq \alpha$ ), and, $x(t)$, after at most one local minimum, increases without bound (i.e. treatment fails, but biomarker may show an early false decline).

(D2) $z(t)$ first decreases to a minimum value $z_{\min }<z_{1}$ and then increases monotonically to $z_{1}$ (if $\left.k_{z}>r\left(1-\left(z_{0} / z_{1}\right)\right)\right)$ and $y(t)$ increases without bound (if $k_{y} \leq \alpha$ ), while $x(t)$ has at most one local maximum and at most one local minimum (in that order) before then increasing without bound (i.e. a drop in normal cells may cause limited early biomarker fluctuations that are misleading).

(D3) $z(t)$ first decreases to a minimum value $z_{\min }<z_{1}$ and then increases monotonically to $z_{1}$ (if $k_{z}>r\left(1-\left(z_{0} / z_{1}\right)\right)$ ) and $y(t)$ decreases to a single local minimum before increasing without bound (if $k_{y}>\alpha$ ). The behavior of $x(t)$ ends in a period of 
unbounded growth but may first exhibit a number of local maxima and/or minima (i.e. in the case of temporary or prolonged disease control, there may be an early sequence of inconsistent biomarker signals).

(See Appendix for a proof of the theorem.)

\section{Remarks.}

1. Expressions for normal and diseased tissue: explicit formulas for the mass of normal cells $z(t)$ and disease cells $y(t)$ are given by

$$
z(t)=\frac{z_{0} z_{1} \mathrm{e}^{r t+\left(k_{z} / a\right) \mathrm{e}^{-a t}}}{z_{1} \mathrm{e}^{\left(k_{z} / a\right)}+r z_{0} \int_{0}^{t} \mathrm{e}^{r s+\left(k_{z} / a\right) \mathrm{e}^{-a s} \mathrm{~d} s}}
$$

and

$$
y(t)=y_{0} \mathrm{e}^{\alpha t-\left(k_{y} / a\right)\left(1-\mathrm{e}^{-a t}\right)} .
$$

2. Expression for biomarker: the biomarker level $x(t)$ is given by an integral expression that involves the transport function $\mu$ :

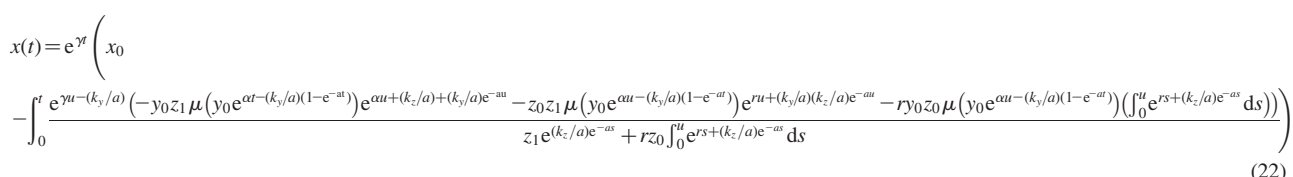

3. Long-term behavior of disease and biomarker: for cases of disease (C and $\mathrm{D})$, while the model does not ever produce an absolutely complete cure (in the sense that the level of disease becomes zero), it may produce disease levels which are undetectably low over a time period comparable to the human lifespan, resulting in a "cure". The time at which an apparently successful treatment ultimately begins to fail is reflected in the long-term behavior of the biomarker, suggesting in some cases a time when re-treatment should be considered. The time at which a relapse in disease levels occurs is $t_{\text {low }}=(1 / a) \ln \left(k_{y} / \alpha\right)$, only realized when $k_{y}>\alpha$.

\section{Model implementation and implications for the use of biomarkers}

In the case (D) of disease and treatment, it is important to recognize the various possibilities that may be observed features of the disease/biomarker dynamics. As seen in cases D1 and D2, a clear case of treatment failure may still allow the possibility of a temporary decline in biomarker levels. Clinicians are likely already aware of the possibility of such false signals, with the model confirming that these may result from actual biomarker dynamics or from changes in the level of normal cells (as well as from spurious test results).

In some cases, there will be a need for retesting often enough to avoid being fooled by the appearance of success in the early biomarker behavior. The fact that biomarker levels may 
make two or more changes of direction before confirming the ultimate direction of disease level suggests that required follow-up, including at least three biomarker readings over an extended period of time (as is practiced in assessing the success of prostate cancer treatment), is prudent. Single-equation models do not generally allow for such a rich collection of behaviors.

Because of the complexity of the general solution (22) for the biomarker $x(t)$ and the many variables and factors contributing to the level of biomarker, we first provide an example of the behavior of this model when $z(t) \equiv z_{0}$ (normal cell volume is steady) and there is no treatment (i.e. $k_{y}=k_{z}=0$ ). In this case (22) simplifies to

$$
x(t)=\mathrm{e}^{-\gamma t}\left(x_{0}+\int_{0}^{t} \mathrm{e}^{\gamma s}\left(\mathrm{e}^{\alpha s} y_{0}+z_{1}\right) \mu\left(y_{0} \mathrm{e}^{a s}\right) \mathrm{d} s\right)
$$

which can be applied with various forms of the transport function $\mu$. As expected in this case, disease and biomarker levels increase exponentially.

Focusing specifically on the case of prostate cancer and PSA, the literature again provides guidance for the assignment of parameter values. In the system model (18)-(20), the value for $\gamma$ comes from the half-life of bound PSA of 2-3 days which yields a value for $\gamma$ of $0.3 /$ day [9]. $z_{0}$ is the average initial prostate size and we choose values for the volume of a normal prostate in the range of $30-45 \mathrm{~cm}^{3}$ [24]. $y_{0}$ is the initial cancerous tumor size and it has been observed that tumor sizes at different stages in cancer can range from 0.19 to $16.9 \mathrm{~cm}^{3}$ with a median average of $2.4 \mathrm{~cm}^{3}$ [10]. At time $t=0$ we are considering this value to be less than $0.19 \mathrm{~cm}^{3}$ because we are looking first at the initial stage of cancer where the tumor is quite small. The initial PSA level will be set equal to 4.3 and the cancer growth rate used is $\alpha=.01$, following the discussion in section 3. The transport function $\mu(y)$, of which the least is known, describes the accelerated leakage of PSA due to increased volumes of cancer cells, although actual measurements of this effect in clinical situations could not be found. The expression for $\mu(\cdot)$ involving the error function, discussed above, may be applicable in many situations, but for this example we will use $\mu(y) \equiv 0.04$. With other parameters chosen as indicated, (23) depicts the expected biomarker behavior in the absence of treatment, revealing a quick jump from 4 to about $5.4 \mathrm{ng} / \mathrm{ml}$, a level which then is held for almost a year before beginning to reflect the cancer's exponential growth.

To illustrate the variety of biomarker behavior that can result from various choices of parameter values in the model (18)-(20), we consider below two additional implementations of the model. The first set of parameter values is given by $\alpha=2, a=.5, k_{y}=5, k_{z}=$ $4, z_{1}=4, z_{0}=8, y_{0}=8, r=2, \gamma=1, x_{1}=40$, and $x_{0}=36$, where the transport function is the increasing function $\mu(w)=10+(10 / 24 \pi)(4 \pi w-\sin (4 \pi w))$. In this case, the nature of the transport function creates a number of maxima and minima for the biomarker given by (22). These undulations are superimposed on a larger-scale pattern which includes a single maximum followed by a single minimum and then followed by the inevitable increase in biomarker levels. While the transport function satisfies all of the conditions of the theorem, it may be seen as an unusual choice because of the ripples in its rising graph. Such behavior, could, however, be related to biorhythms of the host or disease organisms or to some kind of environmental cyclicity.

In a final example, the transport function has a more typical "sinusoidal" pattern, rising from an initial constant value and leveling off at a higher value. The set of parameter values in this case is similar to the set considered previously, differing only in that $r=3.5$, and the 


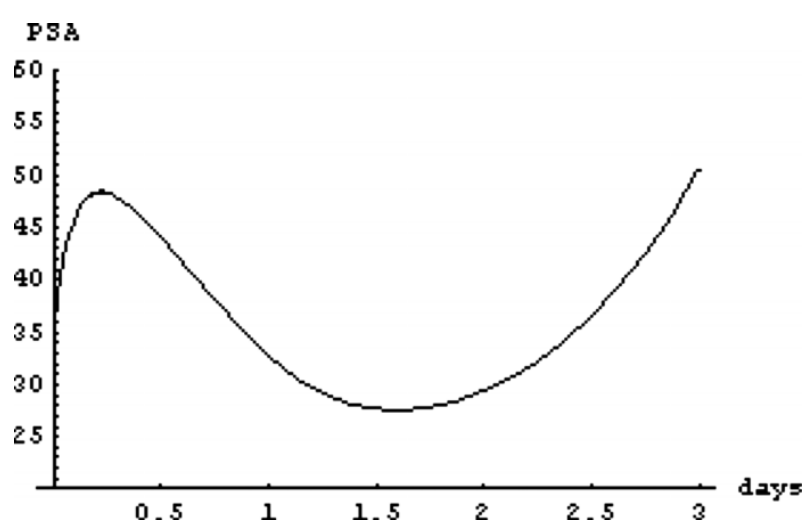

Figure 3. Rapid changes in the direction of biomarker levels demonstrate the possibility of more complex patterns of behavior allowed by the model (18)-(20). See text for additional details.

transport function is

$$
\mu(w)=\left\{\begin{array}{cl}
10+\frac{5}{3 \pi(\pi w-\sin (\pi w))} & \text { if } w \leq 2 \\
\frac{40}{3} & \text { if } w>2
\end{array}\right.
$$

The biomarker behavior in this situation is shown in figure 3 and reveals two reversals in direction, with the second coming quite soon after the first. Clearly the timing of biomarker measurements would be critical in such a case, with an ill-timed sequence of measurements falsely signaling what to expect a short time later. Only regular measurements over a long period of time would allow identification of the complete pattern of biomarker (hence, disease) behavior.

\section{Conclusions and future research}

In its full generality, the model (18)-(20) provides a realistic account of the growth and treatment over time of a disease condition indicated by a biomarker. The implications of the model accommodate the current understanding of biological processes that produce and remove biomarker and regulate its relationship to disease and treatment.

Continued research with the models proposed above, particularly the system model (18)(20), may lead to more accurate simulation of disease/biomarker behavior and correspondingly allow for a better understanding of treatment effectiveness in the case of diseases for which biomarkers exist. Variation of the parameter values in any of the above models may significantly modify the resulting biomarker dynamics. In particular, the possibility of changes in direction for the evolving level of biomarker supports the current practice of frequent testing for PSA and suggests careful monitoring in other biomarker systems. These models might also be adapted for other modes of cancer treatment, e.g. chemotherapy or the use of viruses. In all cases, clinical validation of model dynamics will be important in confirming the usefulness of the models.

Patient data that was considered for this paper was made available from public sources, particularly those referenced in Ref. [19]. Some parameter values used in the models 
described above were selected to demonstrate various possible patterns of behavior and do not necessarily represent parameter sets from a single patient. Further investigation of data on trends of growth and treatment kinetics collected directly from prostate cancer patients would help to achieve a more accurate implementation of these models. Because the parameter values play such a determinate role in the results obtained from each model, reliable estimates of those values will be necessary to establish precise modeling of prostate cancer growth and treatment. In particular, further studies to carefully describe the nature of membrane transport may provide valuable insights regarding the disease process in the case of prostate cancer. Future investigations of other biomarker systems will necessarily rely on the accurate estimation of their corresponding parameter values.

\section{Acknowledgements}

The authors are grateful for the helpful observations and suggestions of the referees.

\section{References}

[1] Craft, D., Wein, L. and Selkoe, D., 2002, A mathematical model of the impact of novel treatements on the A? Burden in the Alzheimer's brain, CSF and plasma, Bulletin of Mathematical Biology, 64, 1011-1031.

[2] Bernard, A.M., 1995, Biokinetics and stability aspects of biomarker: recommendations for application in population studies, Toxicology, 101, 65-71.

[3] Gadduchi, A., et al., 2004, The predictive and prognostic value of serum CA 125 half-life during paclitaxel/platinum-based chemotherapy in patients with advanced ovarian carcinoma, Gynecological Oncology, 93, 131-136.

[4] Brosman, S.A., Prostate-specific antigen, June 2002. [Accessed 14 June 2004]. http://www.emedicine.com/ MED/topic3465.htm

[5] Wu, J. and Nakamura, R., 1997, Human Circulating Tumor Markers (Chicago: American Society of Clinical Pathologist).

[6] Gleave, M., et al., 2004, Prostate-specific antigen as a prognostic predictor for prostate cancer, [Accessed 9 June 2004]. http://www.prostatepointers.org/bruchovsky/np4/paper.html

[7] The Urological Clinics of North America. Prostate Specific Antigen: The Best Prostatic Tumor Marker, 1997, pp. 25.

[8] Kantoff, P., et al., 2002, Prostate Cancer: Principles and Practice (Lippincott Williams \& Wilkins: Philadelphia).

[9] Denis, L., 1999, Textbook of Prostate Cancer (London: Martin Dunitz).

[10] Lepor, H., 2000, Prostatic Disease (Philadelphia: W.B. Saunders Company).

[11] Bostwick, D.G., 1996, Prospective origins of prostate cancer; prostatic intraepithelial neoplasia and atypical adenomatous hyperplasia, Cancer, 78(2), 330-336.

[12] Montironi, R., et al., 1991, Ouantitation of the prostatic intra-epithelial neoplasis. Analysis of the nucleolar size, number and location, Pathology Research and Practice, 187(2-3), 307-314.

[13] Bostwick, D.G. and Foster, C.S., 1998, Pathology of the Prostate (Philadelphia: W.B. Saunders Company).

[14] Coptcoat, M.J., 1996, The Management of Advanced Prostate Cancer (London: Blackwell Science Ltd).

[15] Rosen, I., Liu, H., Childress, N. and Liao, Z., 2005, Interactively exploring optimized treatment plans, International Journal of Radiation Oncology Biology Physics, 61, 570-582.

[16] Crownover, R.L., et al., 1999, The radiobiology and physics of brachytherapy, Hematology/Oncology Clinics of North America, 13, 477-87.

[17] Dayananda, P.W.A., et al., 2004, A stochastic model for prostate-specific antigen levels, Mathematical Biosciences, 190, 113-126.

[18] Swanson, K., et al., 2001, A quantitative model for the dynamics of serum prostate-specific antigen as a marker for cancerous growth, American Journal of Pathology, 158, 2195-2199.

[19] Dayananda, P.W.A., et al., 2003, Prostate cancer: progression of prostate-specific antigen after external beam irradiation, Mathematical Biosciences, 182, 127-134.

[20] Cox, R.S., et al., 1993, Prostate-specific antigen kinetics after external beam irradiation for carcinoma of the prostate, International Journal of Radiation Oncology, 28, 23-31. 
[21] Kaplan, I.D., et al., 1991, Model of prostatic carcinoma tumor kinetics based on prostate-specific antigen levels after radiation therapy, Cancer, 68, 400-404.

[22] Berges, R.R., et al., 1995, Implication of cell kinetic changes during the progression of human prostatic cancer, Clinical Cancer Research, 1(5), 473-480.

[23] Haustermans, et al., 1997, Cell kinetic measurements in prostate cancer, International Journal of Radiation Oncology Biology Physics, 37, 1067-70.

[24] Bosch, J.L., et al., 1994, Parameters of prostate volume and shape in a community-based population of men 55 to 74 years old, Journal of Urology, 152, 1501-1505.

[25] D'Amico, Anthony V. and Hanks, G.E., 1999, Radiotherapeutic Management of Prostate Adenocarcinoma (London: Arnold).

\section{Appendix A: Proof of the theorem on qualitative behavior of disease/biomarker dynamics}

Case $A$. It is evident that $y(t) \equiv 0$ (no disease) and, similar to (21), $z(t)=z_{0} z_{1} / z_{0}+$ $\left(z_{1}-z_{0}\right) \mathrm{e}^{-r t}$, demonstrating the monotonic convergence of any non-constant $z(t)$. For $z(t) \equiv z_{1}$, (18) has a simple solution that reveals the monotone behavior of $x(t)$. For nonconstant $z(t)$, if ever $x^{\prime}(t)=0$ it must be true that $x^{\prime \prime}(t)=\mu(0) z^{\prime}(t)$. Since $z$ is monotonic, $x(t)$ then can have at most one local minimum or one local maximum, depending on whether $z$ is increasing or decreasing, respectively. For example, if $x_{1} \equiv\left(\mu(0) z_{1} / \gamma\right)<x_{0}<\left(\mu(0) z_{0} / \gamma\right)$ so that $z^{\prime}(t)<0$ and $x^{\prime}(0)>0$, it follows that $x(t)$ has one local maximum and then decreases to $x_{1}$.

Case B. Again $y(t) \equiv 0$. In this case, $z^{\prime \prime}(t)=r z^{\prime}\left(1-\left(z / z_{1}\right)\right)-\left(r z z^{\prime} / z_{1}\right)-k_{z} \mathrm{e}^{-a t} z^{\prime}+a k_{z} \mathrm{e}^{-a t} z$, so any critical point of $z$ must be a minimum. If $z^{\prime}(0) \geq 0$, then $z(t)$ increases to $z_{1}$ but if $z^{\prime}(0)=z_{0}\left(r\left(1-\left(z_{0} / z_{1}\right)\right)-k_{z}\right)<0$, then $z(t)$ will experience a local minimum before increasing to $z_{1}$. The only case not immediately clear is for $z(t)>z_{1}$ in which case $z^{\prime}(t)=$ $z(t)\left(r\left(1-\left(z(t) / z_{1}\right)\right)-k_{z} * \mathrm{e}^{-a t}\right)<-z_{1} * k_{z} * \mathrm{e}^{-a t}$ as long as $z(t)>z_{1}$. By integration on the interval $[0, t]$, it follows that $z(t)<z_{0}-z_{1} * k_{z} / 2 a$ for sufficiently large $t$. By repeating the argument a number of times, it is seen that $z(t)$ would eventually fall below $z_{1}$ and find a minimum. Similar to case A, the number and character of critical points of $x(t)$ are restricted to the identified possibilities.

Case C. It is clear that $z(t)$ is constant or monotone, approaching $z_{1}$ (as in Case A) and that $y(t)$ grows exponentially. In this case, $x^{\prime \prime}(t)=-\gamma x^{\prime}(t)+\mu^{\prime}(y(t)) y^{\prime}(t)(y(t)+z(t))+\mu(y(t))\left(y^{\prime}(t)+\right.$ $\left.z^{\prime}(t)\right)$. If $z(t) \equiv z_{1}$, any critical point of $x(t)$ must be a minimum, so there can be at most one such point. (There is such a local minimum if $x(0)$ is sufficiently large.) Since $z^{\prime}(t) \geq 0$ if $z_{0}<z_{1}$, the only possible critical point for $x(t)$ in that case is also a minimum. In the event that $z_{0}>z_{1}$, computation using the explicit formula for $z(t)$ (see Case A) shows that $z^{\prime \prime}(t)>0$ so that $x^{\prime \prime}(t)$ can change signs at most once between consecutive critical points. That is, $x(t)$ could experience at most one local minimum (while $z^{\prime}(t)>0$ ), preceded possibly by one local maximum (while $z^{\prime}(t)<0$ ). That $x(t)$ grows without bound follows from integration of (18) using the facts that $\mu(y(t))$ is bounded below by a positive constant and $y(t)$ grows exponentially (rate $\alpha$ ).

Case D. An expression can be found for $z(t)$ (see Remark 1) and, as in case B, the two possible behaviors for $z(t)$ can be confirmed. The expression for $y(t)$ in Remark 1 also holds in this case, so that $y(t)$ obtains a local minimum if and only if $k_{y}>\alpha$ and is otherwise strictly increasing. In case D1, it follows from (18) to (20) that $y(t)$ and $z(t)$ are increasing, that any 
critical point of $x(t)$ is a minimum and that $x(t)$ eventually grows without bound. For $x_{0}$ sufficiently large, $x^{\prime}(0)<0$ and $x(t)$ actually takes on a local minimum before increasing.

The key observations needed to establish scenario D2 are that $z^{\prime \prime}(t)$ is strictly positive when $z^{\prime}(t) \leq 0$ (which follows from (20) in this case), that once $z^{\prime}(t)$ becomes positive, it remains positive (as in case B2) and that $y(t)$ is positive, increasing and concave up. Depending on the value of $x_{0}, x(t)$ may experience one local maximum (while $z^{\prime}(t)<0$ ) and/or one local minimum before growing without bound.

Case D3 allows a rich class of possible behaviors. For the parameter relationships indicated, it is clear that $z(t)$ and $y(t)$ each have a single minimum. After that, $z(t)$ approaches $z_{1}$ and $y(t)$ grows exponentially. The behavior of the biomarker, $x(t)$, however, may fit many different patterns. Eventually, $x(t)$ will experience unbounded growth in reaction to the growth in $y(t)$. Earlier, though, depending on the nature of the transport function $\mu$ and the initial values of $x_{0}, y_{0}$ and $z_{0}, x(t)$ may undergo a number of sequential maxima and minima. Some of these behaviors are shown in the examples at the end of section 5 . 


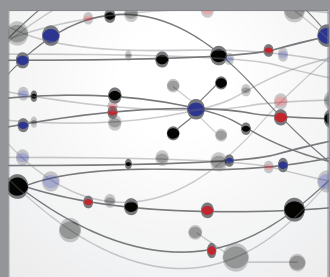

The Scientific World Journal
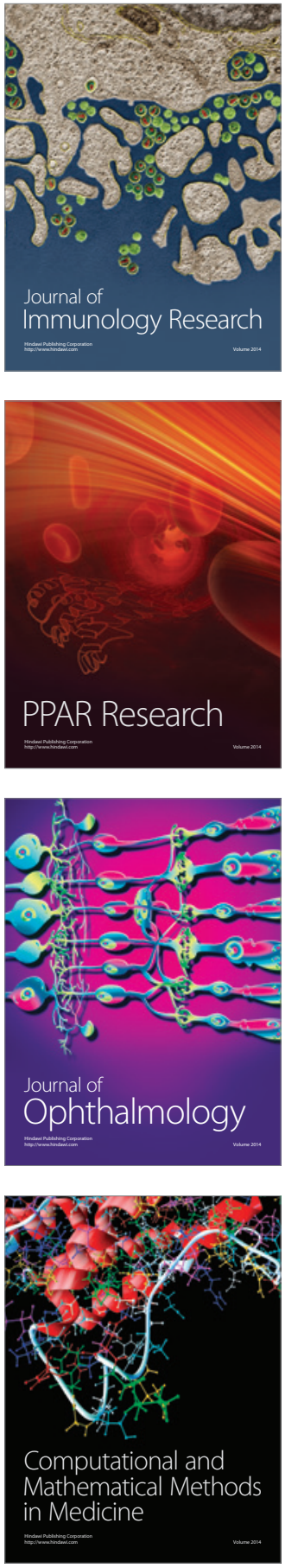

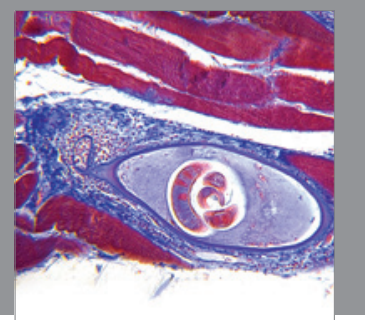

Gastroenterology

Research and Practice
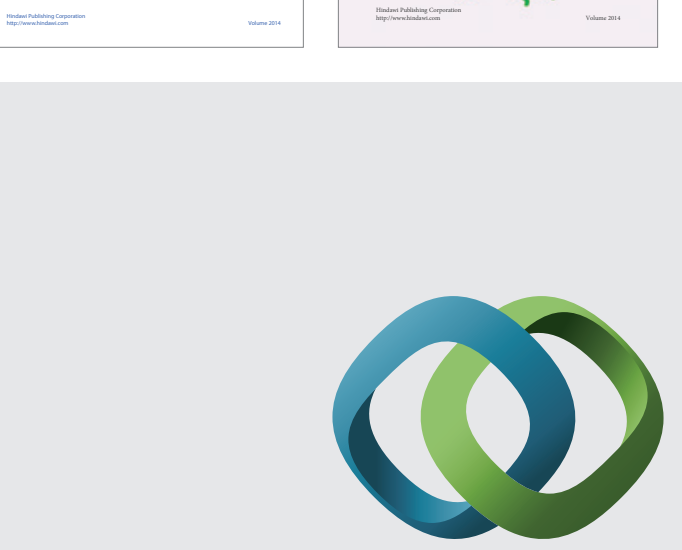

\section{Hindawi}

Submit your manuscripts at

http://www.hindawi.com
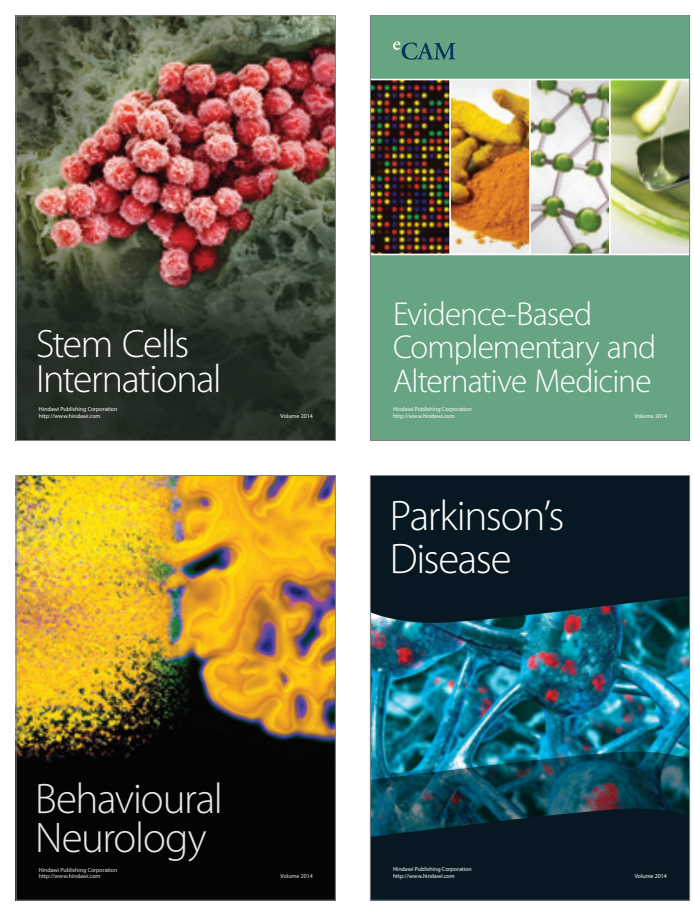

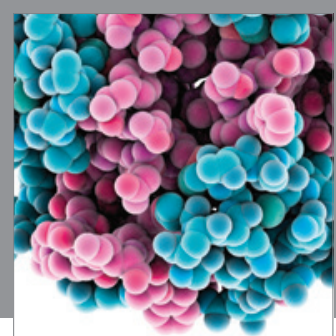

Journal of
Diabetes Research

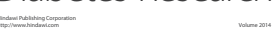

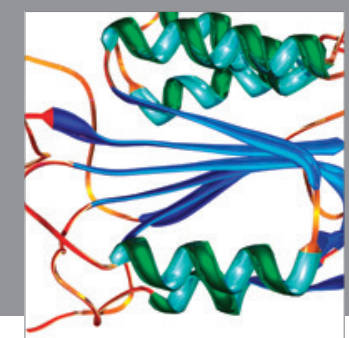

Disease Markers
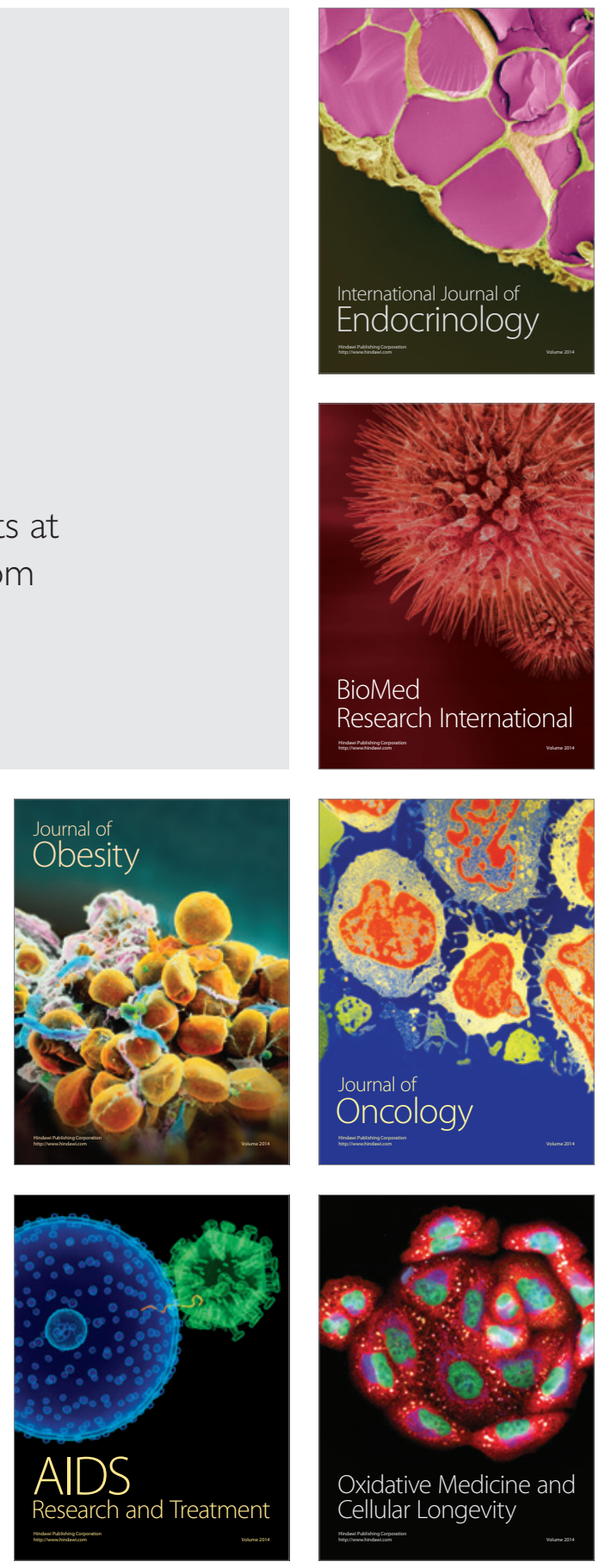